\title{
The calmodulin intergenic spacer as molecular target for characterization of Leishmania species
}

\author{
Aracelis Miranda ${ }^{1}$, Franklyn Samudio ${ }^{1,2}$, Azael Saldaña ${ }^{1}$, Juan Castillo ${ }^{1}$, Adeilton Brandão ${ }^{2}$ and Jose E Calzada ${ }^{\text {** }}$
}

\begin{abstract}
Background: Human leishmaniasis is a neglected disease caused by parasites of the genus Leishmania. Clinical aspects of this disease can vary significantly, reflecting the wide range of parasites in the genus Leishmania. Knowing accurately the Leishmania species infecting humans is important for clinical case management and evaluation of epidemiological risk. Calmodulin is an essential gene in trypanosomatids that modulates the calcium metabolism in various cellular activities. Despite its strong conservation in trypanosomatids, it has been recently observed that its untranslated regions (UTR) diverge among species.

Methods: In this study we analyzed the sequences and the absolute dinucleotide frequency of the intergenic spacer of the calmodulin gene (containing both, $3^{\prime}$ and $5^{\prime} U T R$ ) in nine reference Leishmania species and ten clinical isolates obtained from patients with cutaneous leishmaniasis.

Results: We show that the short calmodulin intergenic spacers exhibit features that make them interesting for applications in molecular characterization and phylogenetic studies of Leishmania. Dendrograms based on sequence alignments and on the dinucleotide frequency indicate that this particular region of calmodulin gene might be useful for species typing between the Leishmania and Viannia subgenera.

Conclusions: Mutations and composition of the calmodulin intergenic spacer from Leishmania species might have taxonomic value as parameters to define if an isolate is identical to a certain species or belongs to one of the two current subgenera.
\end{abstract}

Keywords: Leishmania, Calmodulin, Intergenic spacer, 3'UTR, Phylogenetic analysis, Panama

\section{Background}

Human leishmaniasis is a neglected disease caused by parasites of the genus Leishmania. More than 350 million people are considered at risk of contracting leishmaniasis, and 2 million cases occur yearly in tropical and subtropical countries [1]. Clinical features of this disease can vary significantly, reflecting the wide range of parasites in the genus Leishmania. The sub-genus Viannia is responsible for cutaneous leishmaniasis (CL) in South and Central America, and currently five species are epidemiologically relevant in this region: $L$. V. braziliensis, L. V. panamensis, L. V. peruviana, L. V. guyanensis, L. V. lainsoni, L. V. naiffi $[1,2]$. These species share a number of insect vector and vertebrate hosts, and all of them can cause infections in

\footnotetext{
* Correspondence: jcalzada@gorgas.gob.pa

${ }^{1}$ Instituto Conmemorativo Gorgas de Estudios de Salud, Ciudad de Panamá, Panamá

Full list of author information is available at the end of the article
}

humans that eventually may lead to disease [3]. Knowing accurately the Leishmania species infecting humans is important for clinical case management and evaluation of epidemiological risk. In this sense, several molecular markers have been developed for Leishmania species identification, strain typing, and consequent phylogenetic studies. However, no single genetic marker has been shown to have the sufficient discriminatory power when dealing with closely related Leishmania species. The evaluation of other genetic markers and better analytical tools are needed to completely understand the genetic complexity of Leishmania parasites.

The calmodulin gene is an interesting molecular marker to evaluate. This gene encodes a protein that modulates the interaction of calcium ion with several other proteins in various cellular activities and is a key gene in the metabolism of the cell [4]. In trypanosomes it was first described in T. brucei gambiense [5] and later in T. cruzi [6].
C Biomed Central

(c) 2014 Miranda et al.; licensee BioMed Central Ltd. This is an open access article distributed under the terms of the Creative Commons Attribution License (http://creativecommons.org/licenses/by/2.0), which permits unrestricted use, distribution, and reproduction in any medium, provided the original work is properly cited. 
Interestingly, mutations of calmodulin intergenic spacer have been demonstrated to be specific for T. cruzi major groups [7]. In Leishmania species, after genome sequencing of some species available at TriTrypDB [8], calmodulin appears in the genome at 1-3 copies depending on the species. This tandem arrangement of calmodulin gene has also been observed in $T$. cruzi, and has been deployed in a PCR amplification procedure that yields fragments containing the 3' UTR, intergenic spacer and the 5' UTR [7].

Since the length of the intergenic spacer between calmodulin coding sequences (CDS) varies both intra and inter species, we analyzed only the shortest segment from each Leishmania species.

Additionally, as a tool to supplement information about the molecular taxonomy of Leishmania, we analyzed the composition of dinucleotide frequency from the short calmodulin intergenic spacer to characterize reference strains and clinical isolates of L. panamensis. We show that the overall nucleotide composition of this segment presents features that make them interesting for applications in molecular characterization of Leishmania.

\section{Methods}

Leishmania reference strains used in this study are listed in Table 1. Promastigotes were cultured in Schneider 's insect medium (Sigma Aldrich, Inc., St. Louis, USA) supplemented with $20 \%$ heat-inactivated fetal bovine serum (Gibco, Grand Island, USA) at $26^{\circ} \mathrm{C}$. Total genomic DNA was extracted from promastigotes using a commercial kit (Wizard Genomic DNA Purification Kit, Promega, Madison, WI) according to the manufacturer's instructions. For amplification, oligonucleotides 5utrcal (5' GGAGATC TGCTCGTTGGACA 3') and 3utrcal (5' GGTCAAAT CAACTACGAGGA 3') were designed based on the open reading frame of the calmodulin gene in order to amplify the 3' UTR, the intergenic region and the 5' UTR [7]. PCR was carried out in a $25 \mu \mathrm{L}$ volume containing $12.5 \mu \mathrm{L}$ Master Mix (Promega), 0.4 $\mu \mathrm{mol} / \mathrm{L}$ of each oligonucleotide, $1 \mu \mathrm{L} \mathrm{MgCl}_{2} 25 \mathrm{mM}, 1 \mathrm{ng}$ DNA $(1 \mu \mathrm{L})$ and $9.5 \mu \mathrm{L}$ distilled

Table 1 Leishmania reference strains analyzed in this study

\begin{tabular}{ll}
\hline Species & International code \\
\hline L (Viannia) panamensis & MHOM/PA/98/WR 2306 \\
L (Viannia) peruviana & MHOM/PE/05/WR 2771 \\
L (Viannia) braziliensis & MHOM/PA/02/WR 2355 \\
L (Viannia) braziliensis & MHOM/BR/1975/M2903 \\
L (Viannia) guyanensis & MHOM/BR/1975/M4147 \\
L (Viannia) lainsoni & MHOM/BR/1981/M6426 \\
L (Leishmania) chagasi & MHOM/BR/1974/PP75 \\
L (Leishmania) amazonensis & IFLA/br/1967/PH8 \\
L (Leishmania) mexicana & MHOM/BZ/1982/BEL21 \\
\hline
\end{tabular}

water. Thirty five amplification cycles of $96^{\circ} \mathrm{C}$ for $10 \mathrm{~s}$, $55^{\circ} \mathrm{C}$ for $30 \mathrm{~s}$ and $72^{\circ} \mathrm{C}$ for $30 \mathrm{~s}$, with a final extension at $72^{\circ} \mathrm{C}$ for 7 minutes were carried out. Ten $\mu \mathrm{L}$ of the amplified products were loaded onto $0.8 \%$ agarose gel in $1 \mathrm{X}$ TBE (89 mM Tris borate, $2 \mathrm{mM}$ EDTA [pH 8.3]) and submitted to electrophoresis at $100 \mathrm{~V}$ for $1 \mathrm{~h}$. The gel was stained with Ethidium Bromide and visualized under UV light.

The amplified products were submitted to $1 \%$ low melting point agarose gel in $1 \mathrm{X}$ TBE $(89 \mathrm{mM}$ Tris borate, $2 \mathrm{mM}$ EDTA [pH 8.3]) electrophoresis and the product band was excised from agarose gel and purified using the Qiaquick gel extraction Kit (Qiagen, Chatsworth, Calif) following the manufacturer's instructions. For cloning, the purified PCR products were ligated into the $\mathrm{pCR}^{\mathrm{m}} 4$-TOPO vector using the TOPO TA cloning kit for sequencing (Invitrogen). The ligation product was used to transform JM109 Escherichia coli cells (Promega) following standard protocol [9]. Plasmids were purified with Wizard Plus SV Minipreps (Promega, USA). DNA sequencing of both strands was carried out using BigDye Terminator 3.1 cycle sequencing kit (Applied Biosystems). Primers and Deoxynucleoside Triphosphates were removed using Xterminator kit (Applied Biosystems). The clean sequencing reaction was run through an ABI 3130x sequencer. The chromatograms were edited and aligned with MEGA v.5.0 [10] and Clustal X [11]. Calmodulin intergenic spacer sequences from species listed in Table 2 were obtained from Tritryp database (www.tritrypdb.org). All sequences were retrieved in fasta format and analyzed with software from emboss package (http://emboss.bioinformatics.nl/) as follows: compseq - a) dinucleotide frequency, parameter $=2$.

The output files containing dinucleotide frequency were submitted to webserver www.hiv.lanl.gov/content/ sequence/HEATMAP/heatmap.html for heatmap cluster hierarchical analysis and to PAST [12] for a paired group euclidean distance cluster analysis.

The calmodulin intergenic sequences obtained from Leishmania reference strains were deposited in GeneBankNCBI under accession numbers: JN966910 to JN966919, JQ302012 and JQ302013.

This molecular approach was then employed to analyze ten Leishmania clinical isolates obtained from patients

\section{Table 2 Leishmania species with genome sequences} available at TriTrypdb

\begin{tabular}{ll}
\hline Species & International code/strain \\
\hline L. braziliensis & MHOM/BR/75/M2904 \\
L. donovani & BPK282A1 \\
L. infantum & JPCM5 \\
L. major & strain Friedlin \\
L. mexicana & MHOM/GT/2001/U1103 \\
L. panamensis & MHOM/COL/81/L13 \\
\hline
\end{tabular}




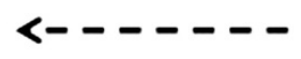

Transcription sense

A

$\begin{array}{lll}\text { Copy } 3 & \text { Copy } 2 & \text { Copy } 1\end{array}$

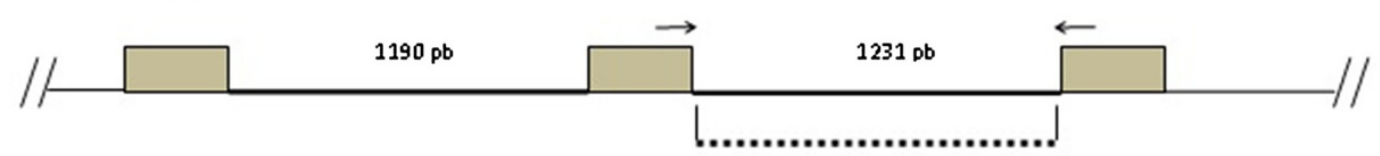

$\square$ ORF Calmodulin

- Intergenic spacer

$\longrightarrow$ Primer 5utrcal

$\longleftarrow$ Primer 3utrcal

...... Amplified and sequenced region

B

Copy 3

Copy 2

Copy 1

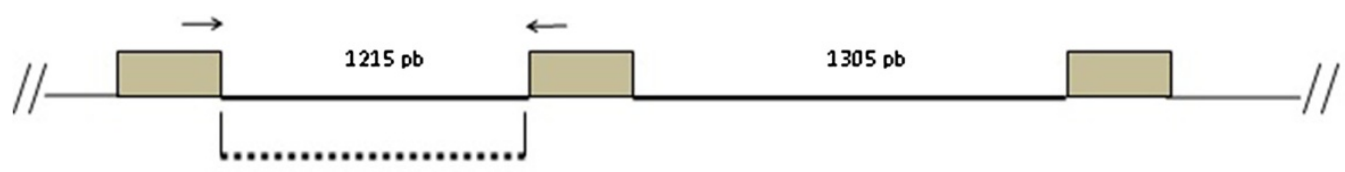

$\square$ ORF Calmodulin

- Intergenic spacer

$\longrightarrow$ Primer 5utrcal

$\longleftarrow$ Primer 3utrcal

....... Amplified and sequenced region

C

Copy $2 \quad$ Copy 1

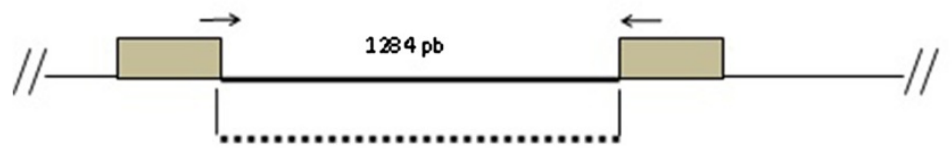

$\square$ ORF Calmodulin

_ Intergenic spacer

$\longrightarrow$ Primer 5utrcal

$\longleftarrow$ Primer 3utrcal

....... Amplified and sequenced region

Figure 1 Calmodulin gene genomic organization. (A) Leishmania braziliensis, (B) Leishmania mexicana, (C) Lesihmania infantum. Arrowheads indicate primer hybridization sites. Amplification proceeds towards intergenic spacer. 
with localized ulcerated lesions who attended the Tropical Medicine Reference Clinic at Gorgas Memorial Institute. Use of these clinical samples was approved by the National Review Board, (Comité Nacional de Bioética de la Investigación, Instituto Conmemorativo Gorgas de Estudios de la Salud, Panama City, Panama).

\section{Ethical approval and consent}

This molecular approach was then employed to analyze ten Leishmania clinical isolates obtained from patients with localized ulcerated lesions who attended the Tropical Medicine Reference Clinic at Gorgas Memorial Institute. Use of these clinical samples was approved by the National Review Board, (Comité Nacional de Bioética de la Investigación, Instituto Conmemorativo Gorgas de Estudios de la Salud, Panama City, Panama). Written informed consent was obtained from the patient for the publication of this report.

\section{Results and discussion}

A graphical summary and the PCR amplification strategy of the calmodulin locus based on genome public sequences from three Leishmania species (L. braziliensis, FR798983.1; L. mexicana, FR799562.1; L. infantum, FR796441.1) is shown in Figure 1. There is variability in both the calmodulin copy number (2-3 copies) and the length of the intergenic spacer (1,190 to $1,400 \mathrm{bp}$ ) among
Leishmania species (Figure 1). We selected species for this study that have at least two calmodulin copies and analyzed the shortest segment of each species listed on Table 1. After PCR amplification and cloning, we compared their sequences with the ones available at Tritrypdb (Table 2). A simple alignment of these sequences with the ones available in GeneDB, allowed the clustering of all studied species into the two currently accepted sub-genera, Leishmania and Viannia (Figure 2). This clustering is interesting because due to low evolutionary pressures, intergenic segments may not reflect taxonomic classifications that are grounded on biological and/or clinical parameters, even for a highly conserved gene like calmodulin.

We next used a bioinformatics tool to further evaluate the distinctiveness among these species taking into account the nucleotide composition, regardless of base order and length. We extracted from the whole intergenic segment from each species the absolute frequency of all sixteen dinucleotides. This approach based on the absolute dinucleotide frequency has recently been used to analyze the composition of trypanosomatid kDNA minicircles [13]. Figure 3 displays a graphical result of this analysis. Again, species are clustered according to allocation in subgenus Leishmania or Viannia. A large proportion of the dinucleotide composition of the calmodulin spacer is represented by CC in sub-genus Viannia and by GG in sub-genus Leishmania.

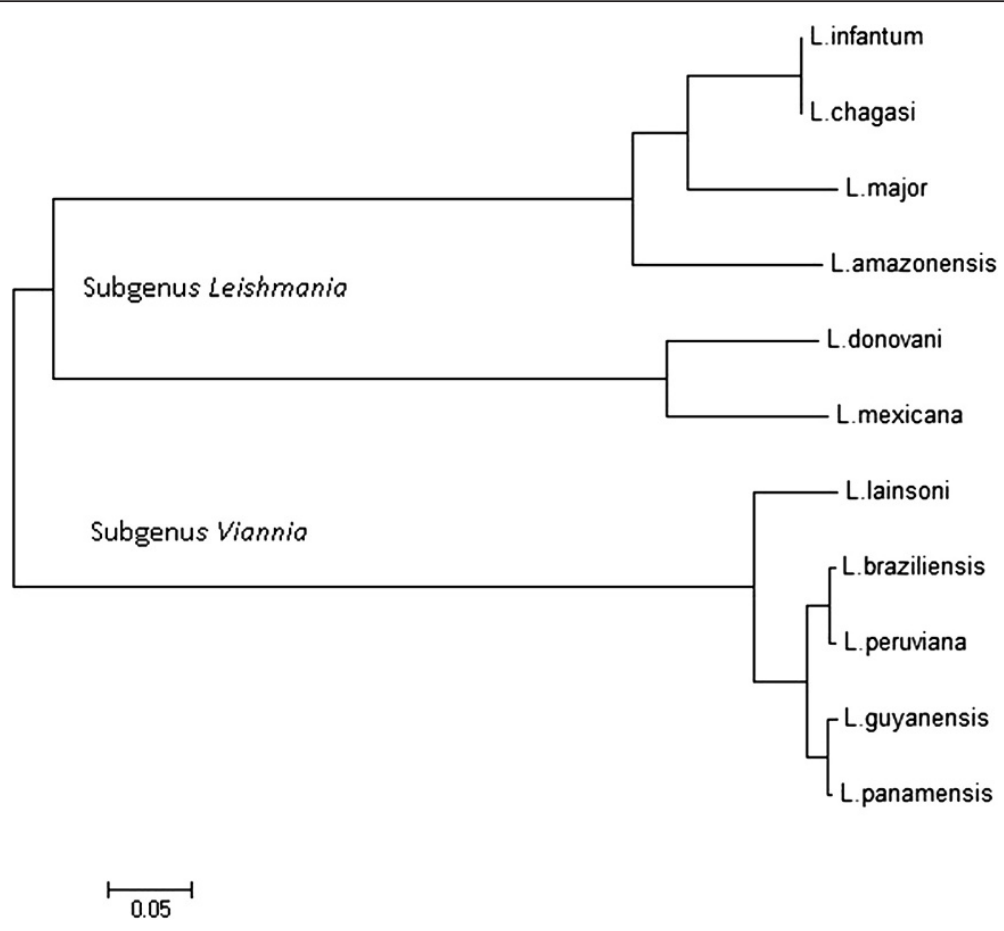

Figure 2 Neighbor-joining phylogeny Leishmania species dendogram of alignment of calmodulin intergenic spacer sequences. Cluster of all studied species into the two currently accepted sub-genera, Leishmania and Viannia. 

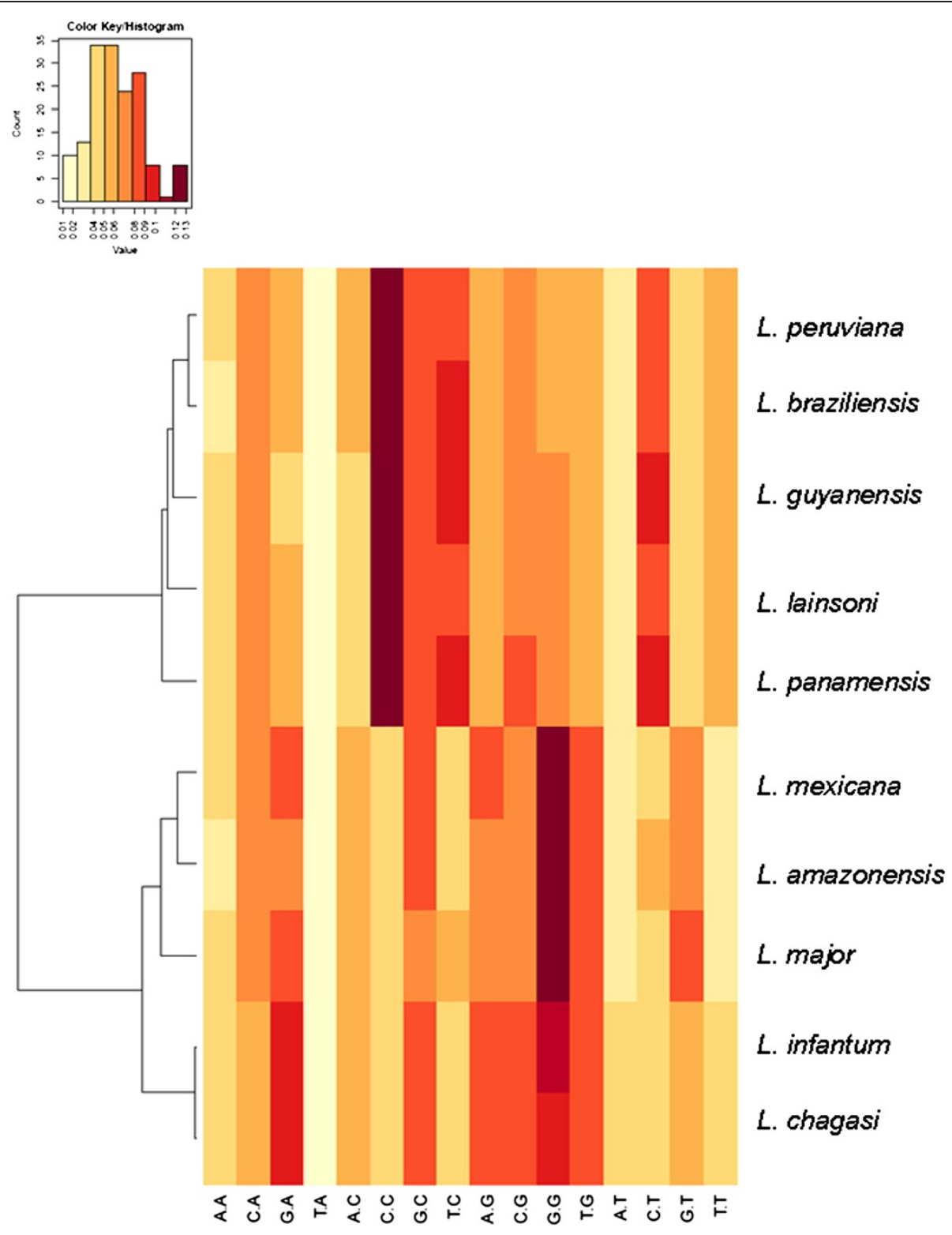

Figure 3 Leishmania species heat map diagram display of the intergenic spacer composition. Is represented by the frequency distribution of the sixteen dinucleotides. Species are clustered according to allocation in subgenus Leishmania or Viannia.

Since this intergenic segment includes both UTRs, ( $3^{\prime}$ and $5^{\prime}$ UTR), we then analyzed the nucleotide composition for each UTR separately. Currently, the genome annotation does not indicate where the $5^{\prime}$ UTR starts or $3^{\prime}$ UTR ends. Thus, we assume an average of 100 and $300 \mathrm{bp}$ for the $5^{\prime}$ and 3' UTR, respectively. Figures 4 and 5 show this compositional analysis expressed in a heatmap. There is some bias to pyrimidine in these probable UTR containing segments, as the compositional picture does not differ from the whole segment analysis, leading to the same dichotomy into two subgenera. Besides confirming the subgenus dichotomy, this method allows a more precise characterization of species as demonstrated by the distinct branch in the distance dendrogram.

In order to deploy the compositional profile of this segment for field applications, we chose the absolute dinucleotide frequency for characterization of $L$. panamensis clinical isolates. As demonstrated in Figure 6, this method worked well for the purpose of species/strain typing, as minor differences in composition allows discrimination of the isolates in comparison to reference strains ( $L$. panamensis 2306 and L. braziliensis 566). These isolates were also characterized at Gorgas Institute as L. panamensis through clinical parameters and biochemical methods. 

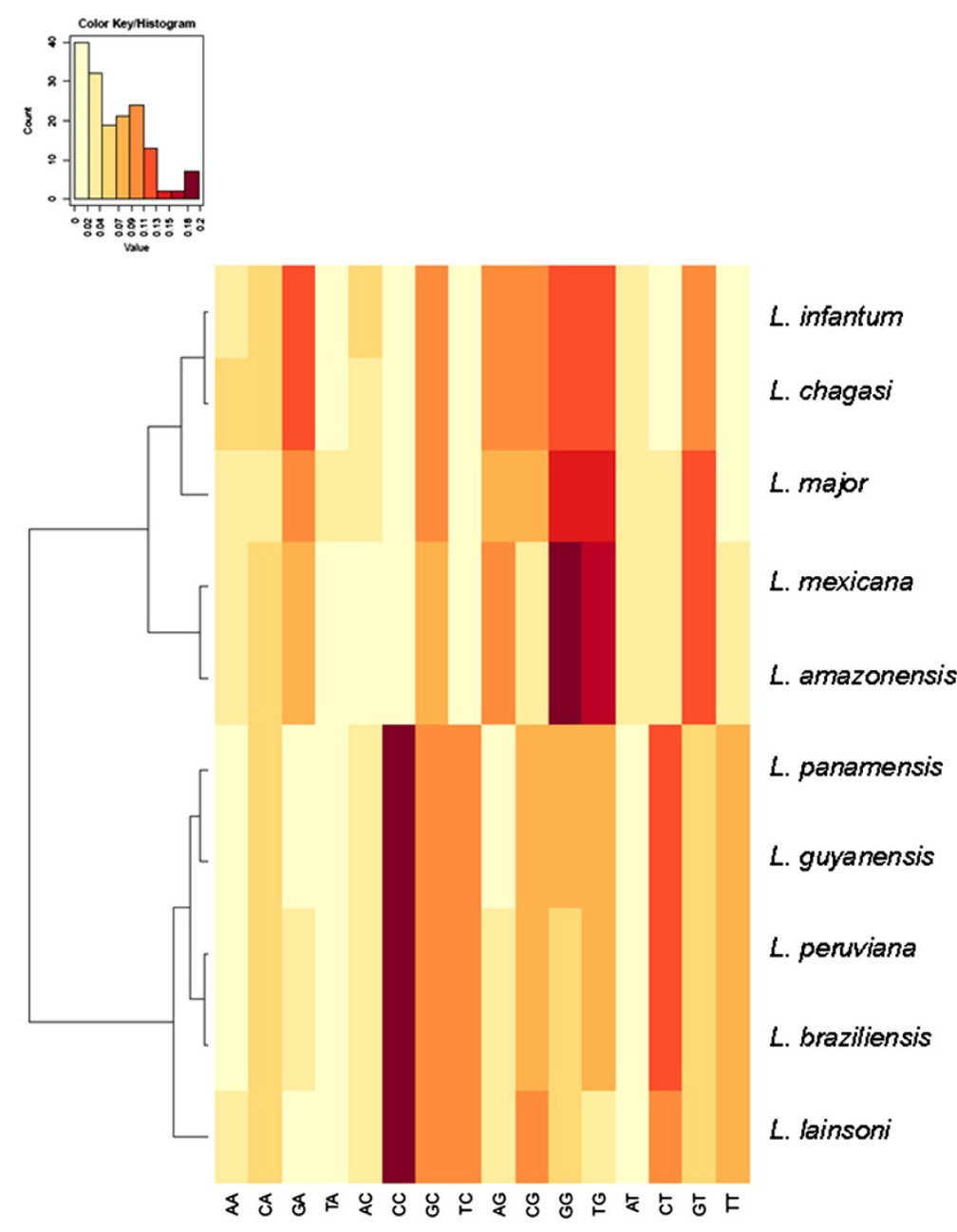

Figure 4 Leishmania species heat map of 5' UTR calmodulin intergenic spacer composition of first $100 \mathrm{bp}$. Species are clustered according to allocation in subgenus Leishmania or Viannia.

The obtained compositional profiles and subsequent cluster analysis confirm that all field isolates belong to L. panamensis.

The Leishmania genus has long been considered a puzzle from the taxonomical point of view. Several clustering schemes based on clinical and biological/biochemical parameters have been proposed to better describe the species spectrum in this genus [14-16]. However, despite enormous progress on the molecular methods, including genomics and proteomics, to shed light on taxonomy and improve the Leishmania species characterization, doubts on the phylogenetic status of certain species still challenge the researchers on this field [17]. Despite the strong conservation of calmodulin gene in trypanosomatids, its UTRs diverge among species. For example, inspection of genome sequences containing the calmodulin $5^{\prime}$ and $3^{\prime}$ UTR from T. brucei are different from the corresponding ones in T. cruzi, L. major and L. braziliensis (a blast search at www.tritrypdb.org shows this fact). However, this divergence is not free of evolutionary or functional pressures because the spacer and UTRs of calmodulin gene are part of a gene class that undergoes polycistronic transcription [18]. As the goal in the investigation of Leishmania species diversity is also to develop markers that can pick up the major transitions in the population structure, calmodulin intergenic segments are good targets for such analysis. Though intraspecies sequence conservation is observed, subgenus Viannia species are clearly distinct from the subgenus Leishmania ones in both length and nucleotide composition. However, taking into account that trypanosomatid gene expression is dependent on post transcriptional processes that are certainly controlled by elements in the $5^{\prime}$ and $3^{\prime}$ UTR and spacer (polypyrimidines region, for instance) [19-21] we decided to inspect these segments with tools based on dinucleotide composition. Mutations and composition of the intergenic segments for some of the Leishmania species analyzed here might have taxonomic value as 

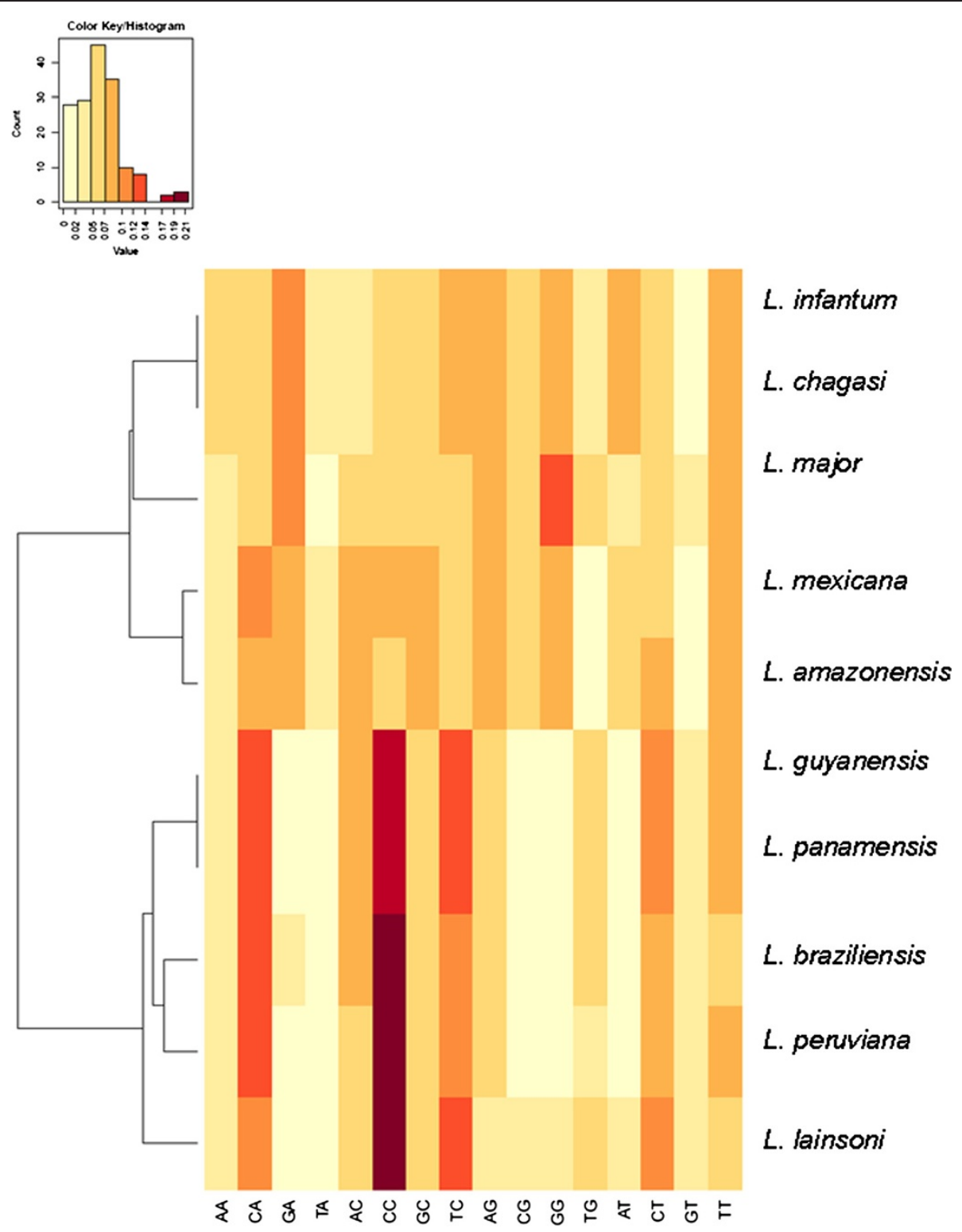

Figure 5 Leishmania species heat map of 3' UTR calmodulin intergenic spacer composition of last $\mathbf{3 0 0}$ bp. Species are clustered according to allocation in subgenus Leishmania or Viannia.

parameters to define if an isolate is identical to a certain species or belongs to one of the two current subgenera. However, for species causing CL, as L. braziliensis, L. peruviana, and L. panamensis sequences are highly similar, casting doubts on the current status of these organisms as unique species.

From an epidemiological point of view it is important to know which Leishmania species circulate in a geographic area. However, from a medical perspective the distinction between Leishmania species causing human infection might be more relevant because the choice of treatment strategy is based on geographical location and mainly in the infecting species [22]. This is the case of L. braziliensis and L. peruviana. Both species cocirculate in some regions of Peru, but only L. braziliensis can potentially cause severe mucocutaneous complications [23]. We observed several sequence variations in the calmodulin intergenic spacer that allow a distinction between these two closely related species belonging to the Viannia sub-genus (Additional file 1).

Recent pioneering studies have used different regions of the heat-shock protein 70 gene (hsp70) with different molecular approaches (sequencing, RFLP, AFLP) for Leishmania species identification as well as for taxonomic and phylogenetic analysis [24-30]. Indeed, the hsp70 gene is probably the single-locus molecular marker more widely 


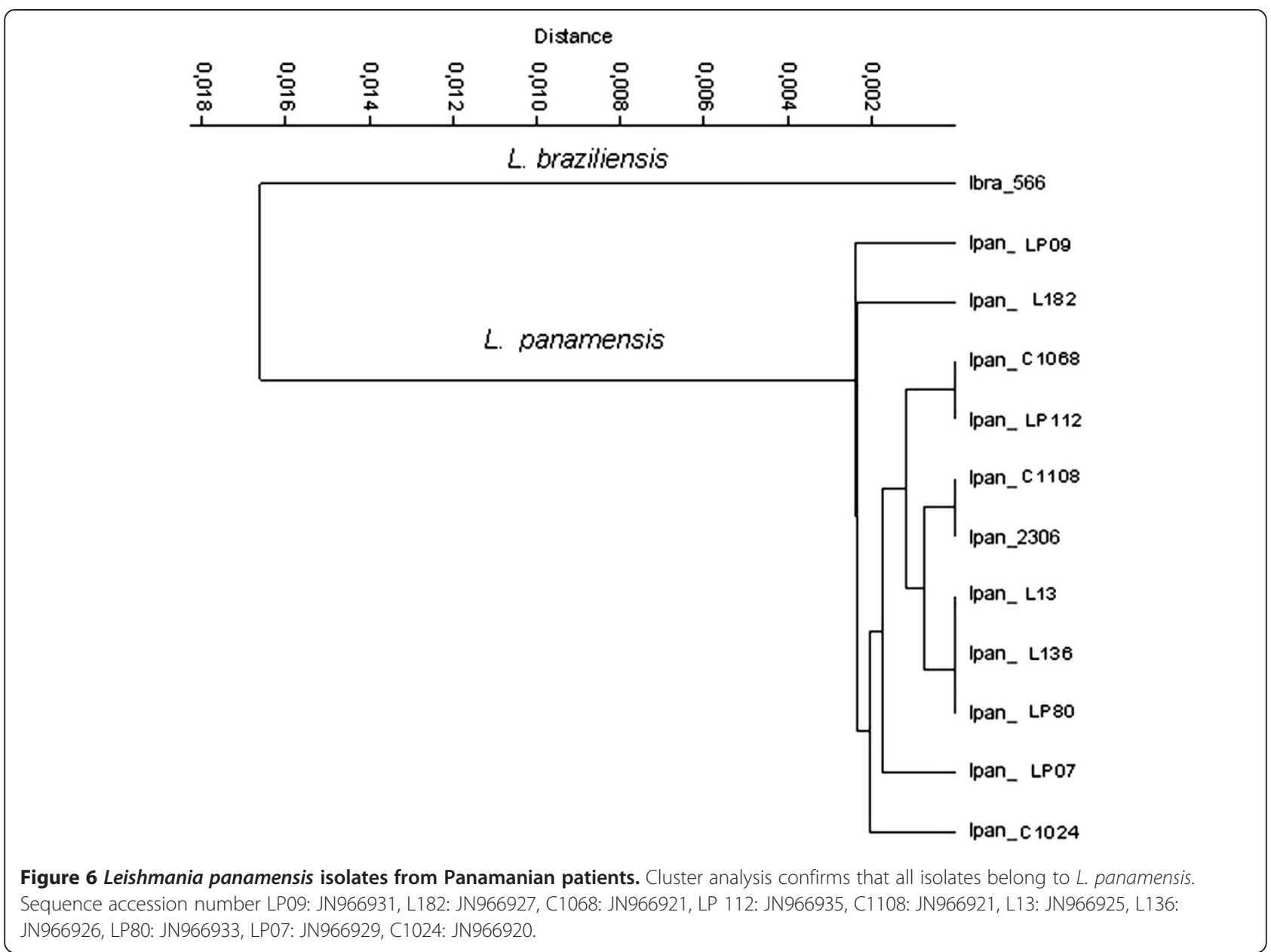

used for identifying/typing Leishmania. In particular, the hsp70/RFLP approach presents several advantages over other molecular methodologies and is now routinely used in many laboratories for these purposes.

In general, the dendrograms we obtained with calmodulin gene agrees with the ones produced using hsp70 as marker. A clear separation between the sub-genus Leishmania and Viannia was observed and nucleotide sequence variations in this segment of the gene made possible to discriminate between Leishmania species/complex (Figures 2, 3,4 and 5 and Additional file 1). However, we recognize that so far we have only evaluated a small number of reference strains and field isolates. In this regard, the real discriminatory power of calmodulin as a marker and its potential phylogenetic inferences still need to be evaluated using a larger number of reference strains and validated with a more field isolates from different geographical areas.

\section{Conclusions}

In conclusion, for the first time we have employed the calmodulin intergenic spacer sequences in a phylogenetic study of Leishmania species. We demonstrated that despite the evolutionary conservation of the calmodulin gene, its intergenic spacer region may be useful to help solve taxonomical questions in the Leishmania genus and also to characterize clinical/field isolates of a particular species.

\section{Additional file}

Additional file 1: Comparison of mutations and their localizations in the calmodulin intergenic spacer of Leishmania Viannia reference strains.

\section{Abbreviations}

CL: Cutaneous leishmaniasis; CDS: Coding DNA sequence; UTR: Untranslated regions.

\section{Competing interests}

All authors declare no competing interests. AS and JEC are members of the Sistema Nacional de Investigación (SNI), SENACYT, Panama.

\section{Authors' contributions}

$A B$, JEC and $A S$ conceived the study and participated in its design and coordination. AM, FS, $A B$ and JC carried out the molecular genetic studies and participated in the sequence alignment and interpretation. $A B, A M$ and JEC drafted the manuscript. All authors read and approved the final manuscript. 


\section{Acknowledgements}

We acknowledge with appreciation the technical assistance of Kadir González, as well as, all members of the Parasitology Department at the Gorgas Institute. This investigation received administrative and financial support from Gorgas Institute and from SENACYT, Panama (grant COL08-080).

\section{Author details}

${ }^{1}$ Instituto Conmemorativo Gorgas de Estudios de Salud, Ciudad de Panamá, Panamá. 'Laboratorio Interdisciplinar de Pesquisas Médicas, Instituto Oswaldo Cruz, Fiocruz, Rio de Janeiro, Brasil.

Received: 26 August 2013 Accepted: 14 January 2014

Published: 19 January 2014

\section{References}

1. World Health Organization: Technical Report Series on the control of the leishmaniasis. N949. Geneva: World Health Organization; 2010.

2. Tsukayama P, Lucas C, Bacon DJ: Typing of four genetic loci discriminates among closely related species of New World Leishmania. Int J Parasitol 2009, 39:355-362.

3. De Brito ME, Andrade MS, Dantas-Torres F, Rodrigues EH, Cavalcanti Mde P, De Almeida AM, Brandão-Filho SP: Cutaneous leishmaniasis in northeastern Brazil: a critical appraisal of studies conducted in State of Pernambuco. Rev Soc Bras Med Trop 2012, 45:425-429.

4. Hoeflich KP, Ikura M: Calmodulin in action: diversity in target recognition and activation mechanisms. Cell 2002, 108:739-742.

5. Tschudi C, Young AS, Ruben L, Patton CL, Richards FF: Calmodulin genes in trypanosomes are tandemly repeated and produce multiple mRNAs with a common 5' leader sequence. Proc Natl Acad Sci 1985, 82:3998-4002.

6. Chung SH, Swindle J: Linkage of the calmodulin and ubiquitin loci in Trypanosoma cruzi. Nucleic Acids Res 1990, 18:4561-4569.

7. Brandao A, Fernandes O: Trypanosoma cruzi: mutations in the $3^{\prime}$ untranslated region of calmodulin gene are specific for lineages $T$. cruzi I, T. cruzi II, and the Zymodeme III isolates. Exp Parasitol 2006, 112:247-252.

8. Aslett $\mathrm{M}$, et al: TriTrypDB: a functional genomic resource for the Trypanosomatidae. Nucleic Acids Res 2010, 38:D457-D462.

9. Sambrook J, Fritschi EF, Maniatis T: Molecular cloning: a laboratorymanual. New York: Cold Spring Harbor Laboratory Press; 1989.

10. Tamura K, Peterson D, Peterson N, Stecher G, Nei M, Kumar S: MEGA 5 : Molecular Evolutionary Genetics Analysis using maximun likelihood, evolutionary distance, and maximun parsimony methods. Mol Bio and Evol 2011, 28:2731-2739

11. Thompson JD, Gibson TJ, Plewniak F, Jeanmougin F, DG DGH: The ClustalX windows interface: flexible strategies for multiple sequence alignment aided by quality analysis tools. Nucleic Acids Res 1997, 25:4876-4882.

12. Hammer O, Harper DAT, Ryan PD: PAST: Paleontological Statistics Software Package for Education and Data Analysis. Palaeontol Electron 2001, 4:9.

13. De Oliveira RPL, Brandão A: An analysis of trypanosomatids kDNA minicircle by absolute dinucleotide frequency. Parasitol Int 2013, 62:397-403

14. Lukes J, Mauricio IL, Schönian G, Dujardin J-C, Soteriadou K, Dedet J-P, et al: Evolutionary and geographical history of the Leishmania Donovani complex with a revision of current taxonomy. Proc Natl Acad Sci U S A 2007, 104:9375-9380.

15. Schönian G, Mauricio I, Gramiccia M, Cañavate C, Boelaert M, Dujardin J-C: Leishmaniasis in the Mediterranean in the era of molecular epidemiology. Trends Parasitol 2008, 24:135-142.

16. Schönian G, Kuhls K, Mauricio IL: Molecular approaches for a better understanding of the epidemiology and population genetics of Leishmania. Parasitology 2011, 138:405-425.

17. Schönian G, Mauricio I, Cupolillo E: Is it time to revise the nomenclature of Leishmania? Trends Parasitol 2010, 26:466-469.

18. Tschudi C, Ullu E: Polygene transcripts are precursors to calmodulin mRNAs in trypanosomes. EMBO J 1988, 7:455-463.

19. Benz C, Nilsson D, Andersson B, Clayton C, Guilbride DL: Messenger RNA processing sites in Trypanosoma brucei. Mol Biochem Parasitol 2005, 143:125-134.

20. Gopal S, Awadalla S, Gaasterland T, Cross GA: A computational investigation of kinetoplastid trans-splicing. Genome Biol 2005, 6:R95.
21. Matthews KR, Tschudi C, Ullu E: A common pyrimidine-rich motif governs trans-splicing and polyadenylation of tubulin polycistronic pre-mRNA in trypanosomes. Genes Dev 1994, 8:491-501.

22. Arevalo J, Ramirez L, Adaui V, et al: Influence of Leishmania (Viannia) species on the response to antimonial treatment in patients with American tegumentary leishmaniasis. J Infect Dis 2007, 195:1846-1851.

23. Nolder D, Roncal N, Davies CR, Llanos-Cuentas A, Miles MA: Multiple hybrid genotipes of Leishmania (Viannia) in a focus of mucocutaneous leishmaniasis. Am J Trop Med Hyg 2007, 76:573-578.

24. Da Silva LA, De Sousa CS, Da Graça GC, Porrozzi R, Cupolillo E: Sequence analysis and PCR-RFLP profiling of the hsp70 gene as a valuable tool for identifying Leishmania species associated with human leishmaniasis in Brazil. Infect Genet Evol 2010, 10:77-83.

25. Fraga J, Montalvo AM, De Doncker S, Dujardin JC, Van der Auwera G: Phylogeny of Leishmania species based on the heat-shock protein 70 gene. Infect Genet Evol 2010, 10:238-245.

26. Ramírez CA, Requena JM, Puerta CJ: Identification of the HSP70-II gene in Leishmania braziliensis HSP70 locus: genomic organization and UTRs characterization. Parasit Vectors 2011, 26(4):1-11.

27. Fraga J, Veland N, Montalvo AM, Praet N, Boggild AK, Valencia BM, Arévalo J, Llanos-Cuentas A, Dujardin JC, Van der Auwera G: Accurate and rapid species typing from cutaneous and mucocutaneous leishmaniasis lesions of the New World. Diagn Microbiol Infect Dis 2012, 74:142-150.

28. Odiwuor S, Veland N, Maes I, Arévalo J, Dujardin JC, Van der Auwera G Evolution of the Leishmania braziliensis species complex from amplified fragment length polymorphisms, and clinical implications. Infect Genet Evol 2012, 12:1994-2002.

29. Requena JM, Chicharro C, García L, Parrado R, Puerta CJ, Cañavate C Sequence analysis of the $3^{\prime}$-untranslated region of HSP70 (type I) genes in the genus Leishmania: its usefulness as a molecular marker for species identification. Parasit Vectors 2012, 5:87.

30. Van der Auwera G, Maes I, De Doncker S, Ravel C, Cnops L, Van Esbroeck M, Van Gompel A, Clerinx J, Dujardin JC: Heat-shock protein 70 gene sequencing for Leishmania species typing in European tropical infectious disease clinics. Euro Surveill 2013, 18:20543.

doi:10.1186/1756-3305-7-35

Cite this article as: Miranda et al:: The calmodulin intergenic spacer as molecular target for characterization of Leishmania species. Parasites \& Vectors 2014 7:35.

\section{Submit your next manuscript to BioMed Central and take full advantage of:}

- Convenient online submission

- Thorough peer review

- No space constraints or color figure charges

- Immediate publication on acceptance

- Inclusion in PubMed, CAS, Scopus and Google Scholar

- Research which is freely available for redistribution 